Supporting Information for:

\title{
Interpolating Nonadiabatic Molecular Dynamics Hamiltonian with Inverse Fast Fourier Transform
}

\author{
Bipeng Wang, ${ }^{1}$ Weibin Chu, ${ }^{2}$ Oleg V. Prezhdo ${ }^{1,2, *}$
}

${ }^{1}$ Department of Chemical Engineering, University of Southern California, Los Angeles, CA 90089, USA

${ }^{2}$ Department of Chemistry, University of Southern California, Los Angeles, CA 90089, USA
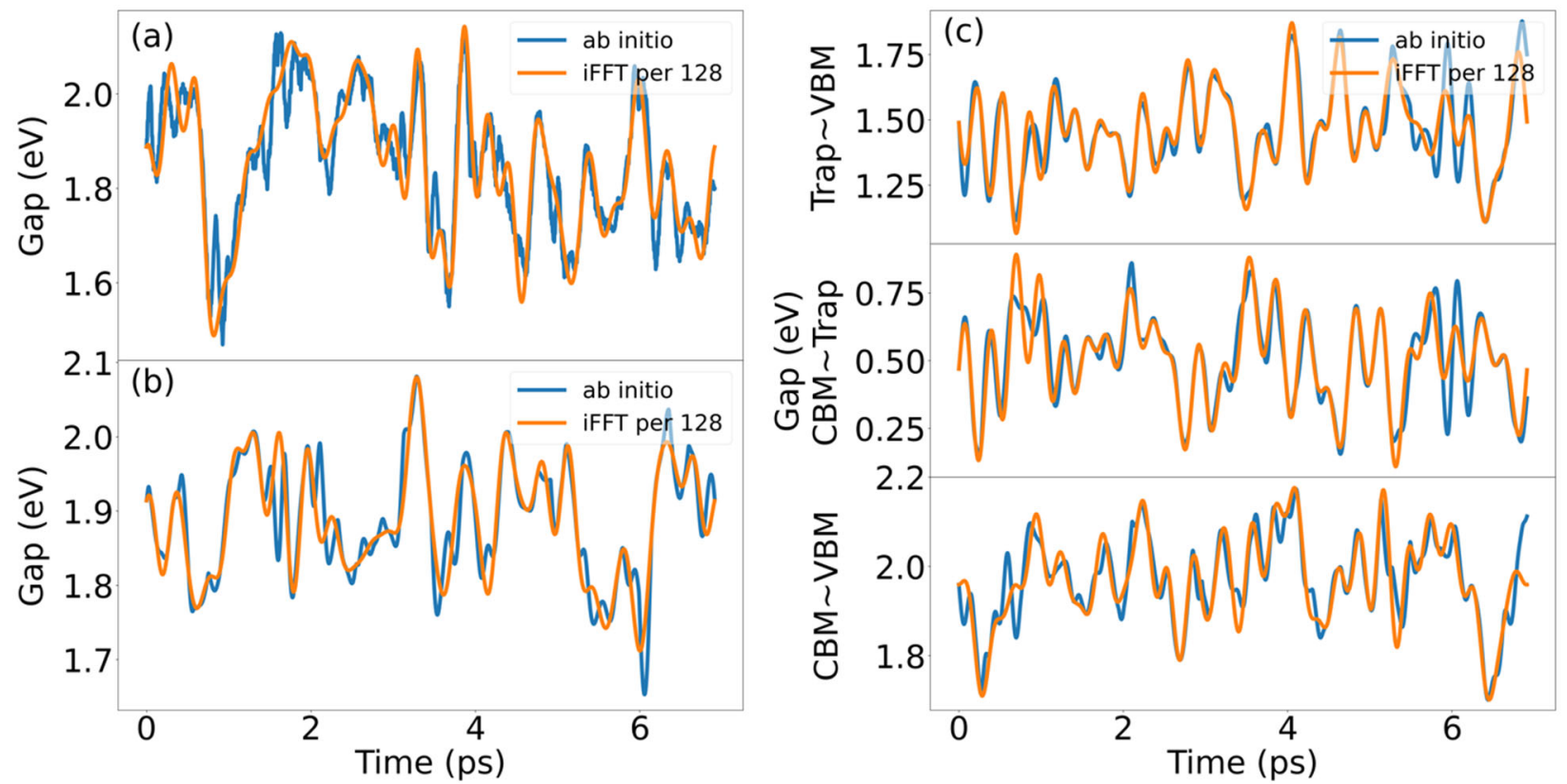

Figure S1. Energy gaps of (a) pristine $\mathrm{MAPbI}_{3}$, (b) pristine $\mathrm{CsPbI}_{3}$, and (c) defective $\mathrm{CsPbI}_{3}$ predicted using the iFFT method. The data used to predict the entire trajectories are selected evenly every 128 points $(0.781 \%, 54$ points in total $)$ from the ab initio data. 

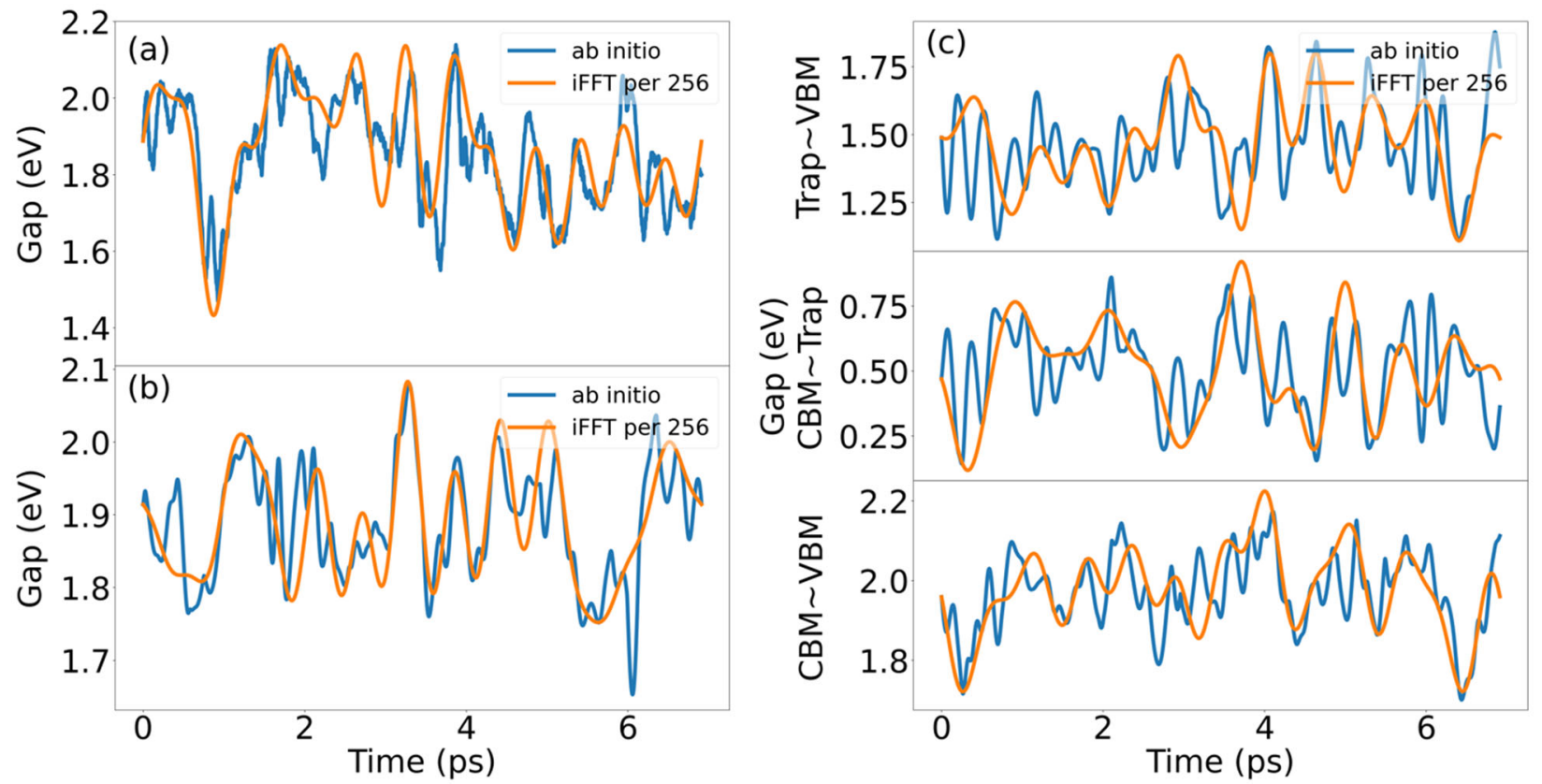

Figure S2. Energy gaps of (a) pristine $\mathrm{MAPbI}_{3}$, (b) pristine $\mathrm{CsPbI}_{3}$, and (c) defective $\mathrm{CsPb}_{3}$ predicted using the iFFT method. The data used to predict the entire trajectories are selected evenly every 256 points $(0.391 \%, 27$ points in total $)$ from the ab initio data.
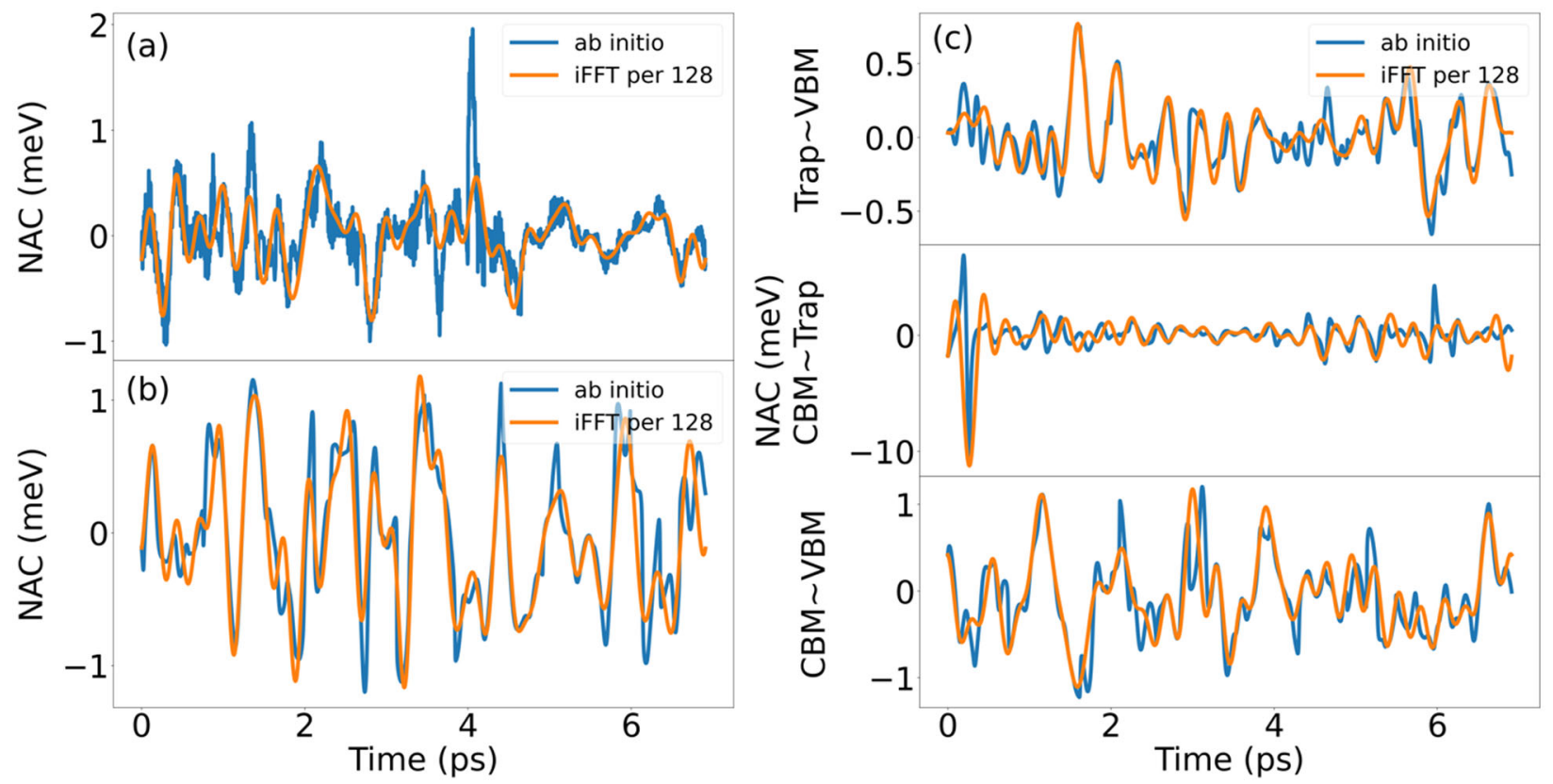

Figure S3. NACs of (a) pristine $\mathrm{MAPbI}_{3}$, (b) pristine $\mathrm{CsPbI}_{3}$, and (c) defective $\mathrm{CsPbI}_{3}$ predicted using the iFFT method. The data used to predict the entire trajectories are selected evenly every 128 points $(0.781 \%, 54$ points in total) from the ab initio data. 

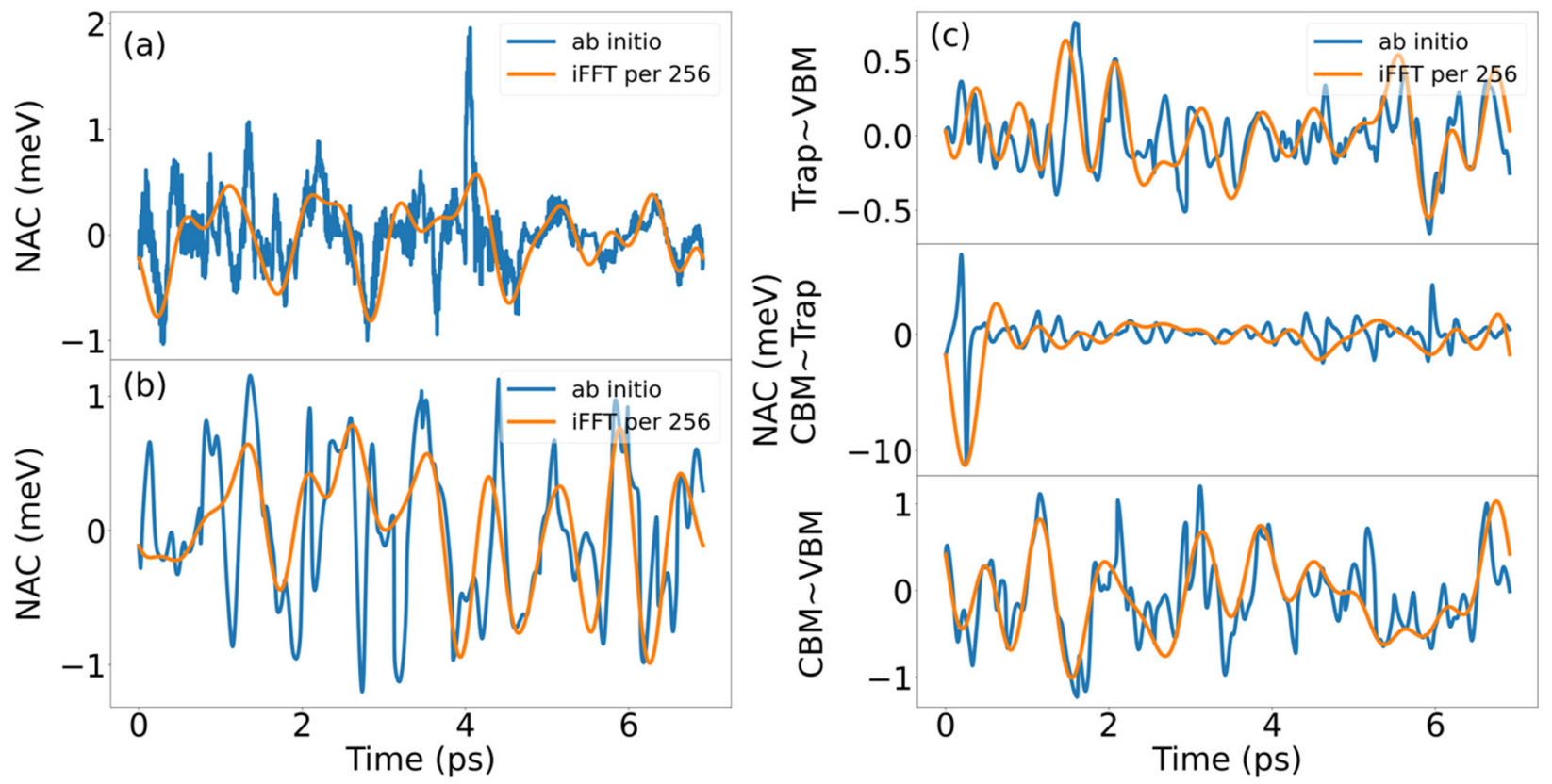

Figure S4. NACs of (a) pristine $\mathrm{MAPbI}_{3}$, (b) pristine $\mathrm{CsPbI}_{3}$, and (c) defective $\mathrm{CsPbI}_{3}$ predicted using the iFFT method. The data used to predict the entire trajectories are selected evenly every 256 points $(0.391 \%, 27$ points in total) from the ab initio data.
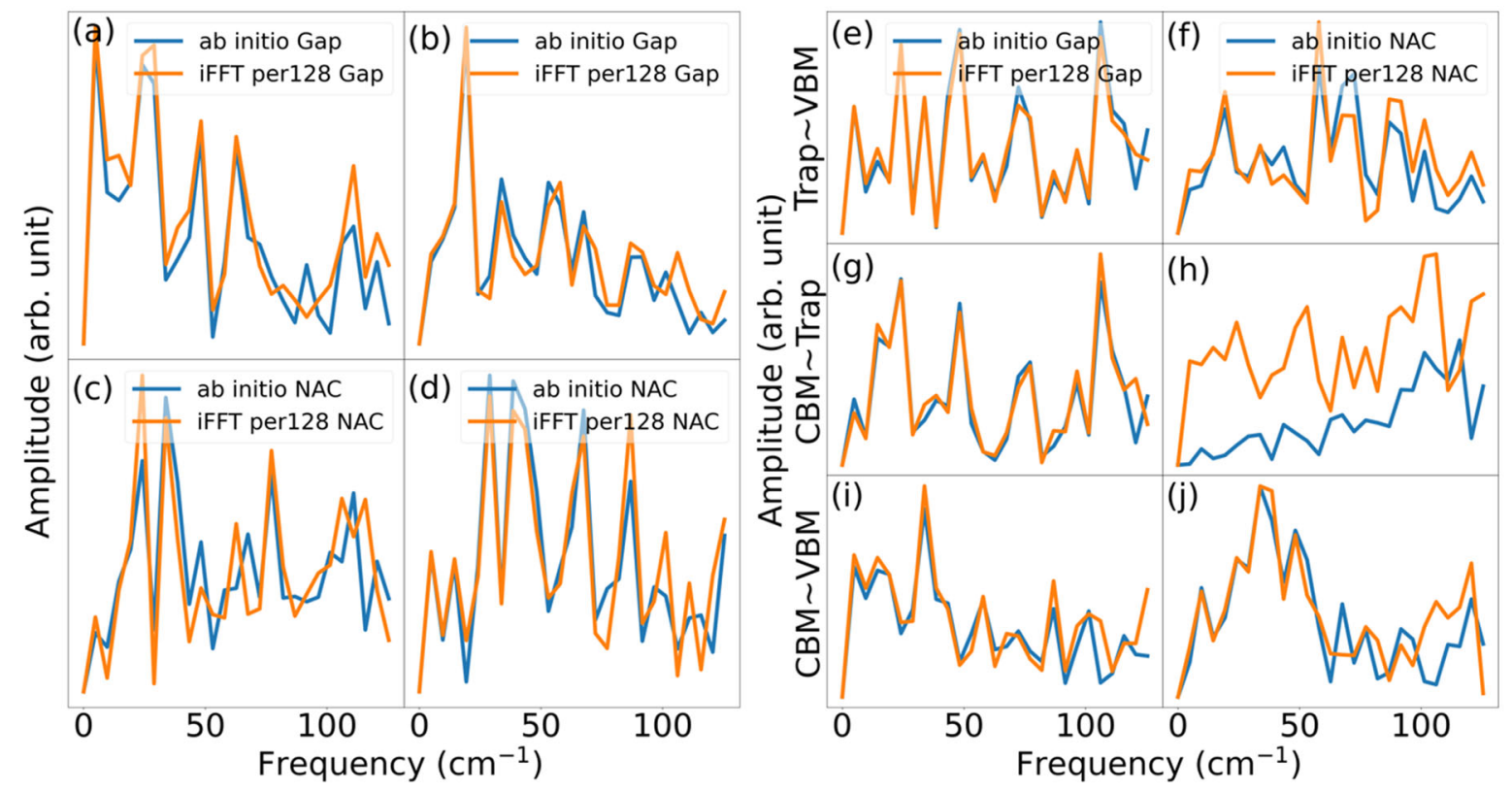

Figure S5. FTs of gap and NAC of (a, c) pristine $\mathrm{MAPbI}_{3},(\mathbf{b}, \mathbf{d})$ pristine $\mathrm{CsPbI}_{3}$ and (e-j) defective $\mathrm{CsPb}_{3}$, corresponding to the data shown in Figures 2 and 3. Every 128 points from the ab initio data are sampled to obtain the iFFT results. 

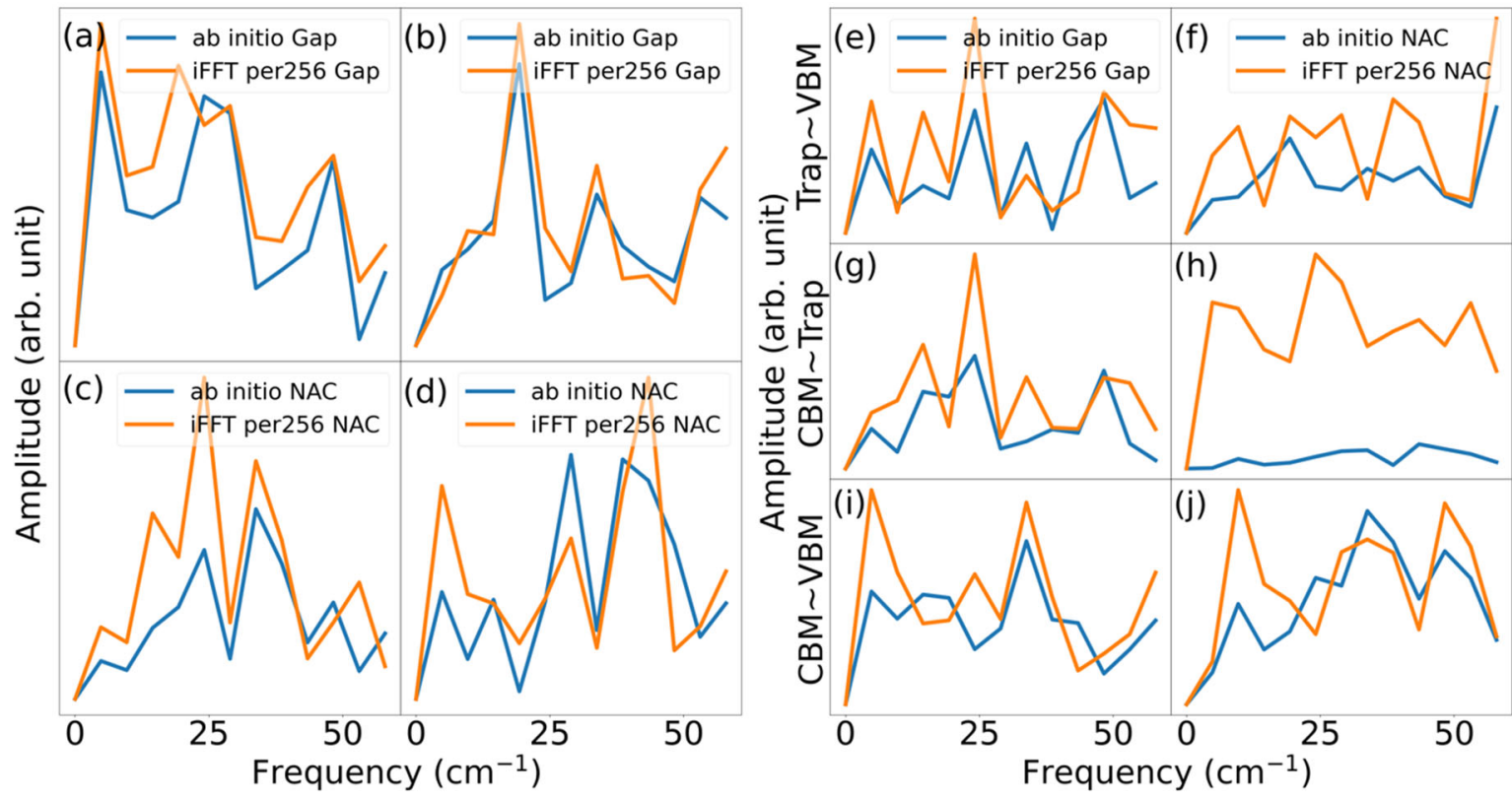

Figure S6. FTs of gap and NAC of (a, c) pristine $\mathrm{MAPbI}_{3},(\mathbf{b}, \mathbf{d})$ pristine $\mathrm{CsPbI}_{3}$ and (e-j) defective $\mathrm{CsPbI}_{3}$, corresponding to the data shown in Figures 2 and 3. Every 256 points from the ab initio data are sampled to obtain the iFFT results.

Table S1. Average Energy Gap, Average Absolute NAC, Root Mean Square (RMS) NAC, PureDephasing Time, and Charge Recombination Time Obtained from the Ab Initio and iFFT Hamiltonian for the Defective $\mathrm{CsPbI}_{3}$ Systems.

\begin{tabular}{|c|c|c|c|c|c|c|c|c|c|c|c|c|}
\hline & $\begin{array}{c}a b \\
\text { initio }\end{array}$ & $\begin{array}{c}\text { iFFT } \\
\text { per } 64\end{array}$ & $\begin{array}{l}\text { iFFT } \\
\text { per } \\
128 \\
\end{array}$ & $\begin{array}{l}\text { iFFT } \\
\text { per } \\
256 \\
\end{array}$ & $\begin{array}{c}a b \\
\text { initio }\end{array}$ & $\begin{array}{c}\text { iFFT } \\
\text { per } \\
64 \\
\end{array}$ & $\begin{array}{c}\text { iFFT } \\
\text { per } \\
128 \\
\end{array}$ & $\begin{array}{c}\text { iFFT } \\
\text { per } \\
256\end{array}$ & $\begin{array}{c}a b \\
\text { initio }\end{array}$ & $\begin{array}{c}\text { iFFT } \\
\text { per } \\
64 \\
\end{array}$ & $\begin{array}{c}\text { ifFT } \\
\text { per } \\
128 \\
\end{array}$ & $\begin{array}{c}\text { iFFT } \\
\text { per } \\
256\end{array}$ \\
\hline & \multicolumn{4}{|c|}{ VBM-Trap } & \multicolumn{4}{|c|}{ CBM-Trap } & \multicolumn{4}{|c|}{ VBM-CBM } \\
\hline Gap (eV) & 1.46 & 1.46 & 1.46 & 1.47 & 0.51 & 0.51 & 0.51 & 0.51 & 1.97 & 1.97 & 1.97 & 1.98 \\
\hline Abs NAC (meV) & 0.17 & 0.17 & 0.17 & 0.20 & 0.66 & 0.81 & 0.92 & 1.19 & 0.37 & 0.38 & 0.35 & 0.37 \\
\hline RMS NAC (meV) & 0.22 & 0.22 & 0.22 & 0.25 & 1.17 & 1.49 & 1.68 & 2.28 & 0.46 & 0.48 & 0.45 & 0.45 \\
\hline $\begin{array}{c}\text { Pure-Dephasing } \\
\text { Time (fs) }\end{array}$ & 4.11 & 4.14 & 4.33 & 4.08 & 4.11 & 4.11 & 4.13 & 3.61 & 6.98 & 7.00 & 6.62 & 6.20 \\
\hline $\begin{array}{c}\text { Transition Time } \\
\text { (ns) }\end{array}$ & 88.26 & 88.48 & 90.45 & 97.26 & 0.10 & 0.07 & 0.05 & 0.03 & & & & \\
\hline
\end{tabular}

